\title{
PREVENTION OF RhD ALLOIMMUNIZATION IN RhD NEGATIVE WOMEN
}

\author{
Marek Lubusky, b* \\ ${ }^{a}$ Department of Obstetrics and Gynecology, Palacky University, University Hospital, Olomouc, Czech Republic \\ ${ }^{b}$ Department of Medical Genetics and Fetal Medicine, Palacky University, University Hospital, Olomouc, Czech Republic \\ E-mail:marek@lubusky.com
}

Received: October 12, 2009; Accepted (with revision): March 1, 2010

Key words: Anti-D immunoglobulin/RhD alloimmunization/Fetomaternal hemorrhage

Background. Despite the introduction of anti-D prophylaxis into clinical practice, $\mathrm{RhD}$ alloimmunization still presents a problem to date. The actual incidence of $\mathrm{RhD}$ alloimmunization in pregnant women remains unknown in most countries. Anti-D immunoglobulin is administered to RhD negative women at a fixed dose and in much greater amounts than is actually necessary. On the other hand, it is not possible to diagnose cases where greater doses are needed. To optimize the prevention of $\mathrm{RhD}$ alloimmunization in $\mathrm{RhD}$ negative women, it is important to diagnose conditions that lead to fetomaternal hemorrhage $(\mathrm{FMH})$, precisely determine the volume and subsequently administer the appropriate dose of anti-D immunoglobulin. The possibility to accurately detect FMH and precisely determine its volume would enable more effective and less costly prevention of RhD alloimmunization. Anti-D immunoglobulin could be administered only in indicated cases and only in doses essentially necessary for prevention of $\mathrm{RhD}$ alloimmunization.

Methods and results. The Cochrane and UpToDate databases of systematic reviews, as well as national guidelines, were reviewed.

Conclusions. Due to the medical significance and indispensable economic costs associated with prevention of RhD alloimmunization, it would be appropriate to establish exact methodical guidelines. The text itself should be limited to a list of potentially sensitising events during which anti-D immunoglobulin should be administered to RhD negative women if anti-D antibodies are not already present. Following each potentially sensitising event, the minimal dose of anti-D immunoglobulin necessary for prevention of RhD alloimmunization should be determined. After 20 weeks of gestation, the volume of FMH should also be determined to specify the necessary dose of anti-D immunoglobulin.

\section{MATERNAL RhD ALLOIMMUNIZATION}

Each person who lacks the red blood cell antigen and is exposed to it will create an antibody. During penetration of $\mathrm{RhD}$ positive fetal erythrocytes into the circulation of an RhD negative mother, the immune system may be stimulated and trigger the creation of antibodies by "alloimmunization". The same immune reaction may also occur during transfusion of antigen-incompatible erythrocytes.

Anti-D antibodies may cause a severe form of haemolytic disease of the fetus and newborn (HDFN Haemolytic disease of the fetus and newborn). Because the $\mathrm{RhD}$ antigen is very potent, even parenteral administration of only $0.1 \mathrm{ml}$ of $\mathrm{RhD}$ positive erythrocytes to $\mathrm{RhD}$ negative individuals will stimulate the production of antibodies. The most common cause of $\mathrm{RhD}$ alloimmunization is hemorrhage, during which fetal erythrocytes enter the mother's circulation.

\section{ABBREVIATIONS}

\footnotetext{
$\mathrm{RhD} \quad$ - rhesus D phenotype (RhD phenotype)

RHD - rhesus D genotype ( $R H D$ genotype)

FMH - fetomaternal hemorrhage

HDFN - haemolytic disease of the fetus and newborn
}

Most cases of RhD alloimmunization however may theoretically be avoided by prophylactic administration of anti-D immunoglobulin in the necessary dose after every potentially sensitising event (Table 1).

\section{THE INCIDENCE OF RhD INCOMPATIBLE PREGNANCIES}

The incidence of RhD incompatibility varies according to race and ethnic background. Approximately $15 \%$ of the Caucasian population is $\mathrm{RhD}$ negative. In most other populations however, the incidence of an $\mathrm{RhD}$ negative phenotype is significantly lower, in African-Americans the incidence is 5-8\%, in Asians and Native Americans 1-2\%. In the Caucasian population, an $\mathrm{RhD}$ negative woman has an $85 \%$ probability that her partner will be $\mathrm{RhD}$ positive, in $60 \%$ heterozygous and in $40 \%$ homozygous at locus $R H D$. In approximately $10 \%$ of all pregnancies, the situation arises where an RhD negative mother will have an RhD positive child and approximately $60 \%$ of $\mathrm{RhD}$ negative women will have an $\mathrm{RhD}$ positive child in their first pregnancy.

In the Czech Republic, if prevention of $\mathrm{RhD}$ alloimmunization was not performed in $\mathrm{RhD}$ negative women following potentially sensitising events, approximately 
2000 women would annually become alloimmunized. Although during the last three decades the introduction of anti-D prophylaxis has led to a decrease in incidence, it remains a problem even today. In the USA in the year 2001, the incidence of $\mathrm{RhD}$ alloimmunization was 6.7 per 1000 liveborn infants ${ }^{1}$.

Such data are not available in the Czech Republic. However, if similar results are assumed, there are about $670 \mathrm{RhD}$ alloimmunized pregnant women annually. If two-thirds of these women will have an RhD positive fetus, we may assume that there will be about 447 at-risk fetuses annually.

\section{PREVENTION OF RhD ALLOIMMUNIZATION IN RhD NEGATIVE WOMEN}

At the beginning of every pregnancy, the woman's AB0 $+\mathrm{RhD}$ blood types are determined and screening for irregular anti-erythrocyte antibodies (from herein referred to only as "anti-erythrocyte antibodies") is performed. In the absence of anti-erythrocyte antibodies at the beginning of pregnancy, a follow-up antibody screening is performed at 28 weeks of gestation in all pregnant women ( $\mathrm{RhD}$ negative and $\mathrm{RhD}$ positive). In $\mathrm{RhD}$ negative women it is performed before antenatal prophylaxis of RhD alloimmunization, and in RhD positive women it is performed due to the possible development of other than anti-D antibodies causing severe haemolytic disease of the newborn.

If the woman's blood type is $R h D^{w}$ positive "weak rhesus positive" (approx. 1\% of those RhD positive), prevention of $R h D$ alloimmunization is generally not indicated.

In cases of weak RhD phenotype "weak D" (formerly labeled $D^{u}$ ), there is quantitative weakening of $D$ antigen expression. All D epitopes are weakly expressed, but individuals do not form anti-D antibodies during contact with erythrocytes with normal $D$ expression.

In contrast, partial $R h D$ phenotypes „partial $D$, variant $D$ “ are $R h D$ positive phenotypes in which some epitopes of the RhD antigen are not expressed. During contact with RhD positive erythrocytes, individuals with partial $D$ phenotype may form antibodies against D epitopes, which are lacking on their erythrocyte surface.

It is necessary to distinguish quantitative weakening of the RhD antigen where it is unnecessary to perform prevention of $R h D$ alloimmunization and qualitative variants of the RhD antigen where prevention is indicated. Individual consultation with the laboratory is necessary! The effectiveness of screening for anti-erythrocyte antibodies at 28 weeks of gestation has not been confirmed but is performed in a number of countries (Europe, USA, Canada, Australia).

The incidence of antepartal RhD alloimmunization is 1-2\%; however, in 90\% of cases it occurs after 28 weeks of gestation ${ }^{9-10}$. The incidence of $R h D$ alloimmunization before 28 weeks is therefore 0.1-0.2\%. Follow-up examination of anti-erythrocyte antibodies in all RhD negative women at 28 weeks of gestation could then enable
Table 1. Events following which anti-D immunoglobulin should be given to all RhD negative women with no anti-D antibodies ${ }^{2-7,19-23}$. Dosage and timing of anti-D immunoglobulin administration ${ }^{6-7,22-23}$.

\section{Delivery of an $\mathrm{RhD}$ positive infant* \\ Abortion}

- therapeutic termination of pregnancy

- spontaneous abortion followed by instrumentation

- spontaneous complete or incomplete abortion after 12 weeks' gestation

- threatened abortion before 12 weeks when bleeding is heavy or repeated or is associated with abdominal pain; in particular, if these events occur as gestation approaches 12 weeks

- threatened abortion after 12 weeks when bleeding continues intermittently, anti-D immunoglobulin should be given at approximately 6-week intervals, and the volume of fetomaternal hemorrhage should be assessed

Invasive prenatal diagnosis

- chorionic villus sampling

- amniocentesis

- cordocentesis

\section{Other intrauterine procedures}

- evacuation of the uterus because of mola hydatiforma

- multifetal reduction

- fetal therapy (insertion of shunts etc.)

\section{Antepartum hemorrhage}

when bleeding continues intermittently, anti-D immunoglobulin should be given at approximately 6 -week intervals, and the volume of fetomaternal hemorrhage should be assessed

\section{External version of the fetus \\ Abdominal trauma \\ Ectopic pregnancy \\ Intrauterine fetal death \\ Stillbirth}

\section{Dose:}

before 20 weeks gestation $50 \mu \mathrm{g}(250 \mathrm{IU})$ after 20 weeks gestation** $100 \mu \mathrm{g}$ (500 IU)

Timing:

as soon as possible, but no later than $\mathbf{7 2}$ hours after the event.

* also if the RhD type of the infant has not been determined or is in doubt

** in conjunction with a test to assess the volume of any fetomaternal hemorrhage 
diagnosis of 10-20 cases of RhD alloimmunization per 100000 deliveries annually. The developed alloimmunization of the mother however does not place the fetus at risk of severe haemolytic disease in the current pregnancy. In such cases, it would therefore not be necessary to administer anti-D immunoglobulin.

Before screening for anti-erythrocyte antibodies in the mother's serum, it is always necessary to specifically inquire whether the woman had already been administered immunoglobulin anti-D (from herein referred to only as "IgG anti-D") during this pregnancy. If so, it is necessary to include this information on the examination requisition slip, because persisting levels of IgG anti-D could falsely lead to suspicion of maternal RhD alloimmunization. Similarly, if during the follow-up screening presence of anti-D antibodies is confirmed, it is best to once more inquire whether IgG anti-D was administered during this pregnancy before a diagnosis of RhD alloimmunization of the mother is established.

The half-life of the administered IgG anti-D is approximately 24 days. In 15-20\% of patients who were administered IgG anti-D at 28 weeks of gestation, it is nonetheless still possible to detect a low titre of anti-D (usually 2 or 4) at the time of labour ${ }^{2}$.

$\mathrm{RhD}$ negative women where the presence of anti-D antibodies in the serum was not confirmed, are administered a dose of $125 \mu \mathrm{g} \mathrm{IgG}$ anti-D intramuscularly at 28 and 34 weeks of gestation. IgG anti-D may also be administered as a single dose of $250 \mu \mathrm{g}$ at 28 weeks of gestation.

This procedure may lead to an $80 \%$ decrease in the incidence of antepartal RhD alloimmunization (from 1\% to $0,2 \%)^{7}$.

In the $1^{\text {st }}$ trimester, women who are $\mathrm{RhD}$ negative are administered $50 \mu \mathrm{g}$ IgG anti-D after spontaneous miscarriage followed by evacuation of the uterus, induced abortion (therapeutic termination of pregnancy), evacuation of the uterus because of mola hydatiforma, chorionic villus sampling or after operation for ectopic pregnancy.

Alloimmunization by the RhD antigen may already be detected at 6 weeks of gestation 4 .

The risk of $R h D$ alloimmunization after spontaneous miscarriage is 1.5-2\%, after induced abortion 4-5\% (ref. ${ }^{2}$ ), after chorionic villus sampling $14 \%$ (ref. $\left.{ }^{11}\right)$.

The total volume of fetal blood at 12 weeks of gestation $=$ $3 \mathrm{ml}$ (FMH 1.5ml), sufficient dose of IgG anti-D = $30 \mu \mathrm{g}$. Therefore up until 12 weeks of gestation, a sufficient dose of $\operatorname{Ig} G$ anti-D for prevention of $D$ alloimmunization is $50 \mu g$.

In the $2^{\text {nd }}$ and $3^{\text {rd }}$ trimester, after induced abortion (therapeutic termination of pregnancy), amniocentesis, cordocentesis, or after other invasive prenatal diagnostic or therapeutic procedures, after antepartum hemorrhaging, intrauterine fetal death, attempt at external cephalic version of a breech presentation, after abdominal trauma or in situations where there is a potential risk of sensitization of the mother by RhD antigens of the fetus, a dose of $50 \mu \mathrm{g}$ of $\mathrm{IgG}$ anti-D is administered to $\mathrm{RhD}$ negative mothers until 20 weeks of gestation, after 20 weeks of gestation $100 \mu \mathrm{g}$ of IgG anti-D are administered. After 20 weeks of gestation, the volume of fetomaternal hemorrhage $(\mathrm{FMH})$ should also be determined to specify dosing.

The risk of $R h D$ alloimmunization after amniocentesis is $2-5 \%$ (ref. ${ }^{3}$ ).

In cases of continued or repeated hemorrhaging after 12 weeks of gestation, $100 \mu \mathrm{g}$ IgG anti-D are repeatedly administered in 6-week intervals, and during each episode of hemorrhaging the volume of fetomaternal hemorrhage (FMH) should be determined to specify the dose of IgG anti-D necessary for prevention of $\mathrm{RhD}$ alloimmunization of the mother ${ }^{2-5}$.

For $\mathrm{RhD}$ negative women, after delivery of an $\mathrm{RhD}$ positive child, if the presence of anti-D antibodies was not detected in the serum, a 50-100 $\mu$ g dose of IgG anti-D must be applied intramuscularly and the volume of FMH should be determined to specify the dose of IgG anti-D necessary to prevent $\mathrm{RhD}$ alloimmunization of the mother. If the FMH volume is not determined, a dose of 200-300 $\mu \mathrm{g}$ is administered. The effectiveness of administering a standard dose of over $100 \mu \mathrm{g}$ to all women has not been demonstrated $^{6,22}$. This dose should be administered immediately following delivery, no later than 72 hours after delivery.

A $10 \mu \mathrm{g}$ dose of IgG anti-D administered intramuscularly should cover $0.5 \mathrm{ml}$ of fetal $R \mathrm{hD}$ positive erythrocytes or $1 \mathrm{ml}$ of whole blood. This means that $125 \mu \mathrm{g}(250 \mu \mathrm{g})$ of IgG anti-D should prevent alloimmunization after fetomaternal hemorrhage of $12.5 \mathrm{ml}(25 \mathrm{ml})$ of whole fetal blood.

In approximately $1.5 \%$ of deliveries is fetomaternal hemorrhage more than $5 \mathrm{ml}$, in only $1 \%$ of deliveries does fetomaternal hemorrhage surpass $12.5 \mathrm{ml}$ and only $0.5 \%$ of deliveries have fetomaternal hemorrhage greater than $25 \mathrm{ml}^{12-15}$. However, in nearly 50\% of all cases no risk factor is present ${ }^{14-16}$.

A number of countries therefore recommend assessing the volume of FMH after delivery to specify the dose of Ig $G$ anti-D necessary to prevent $R h D$ alloimmunization of the mother (Australia, Canada, USA, Great Britain, France, Ireland).

There is a greater risk of fetal erythrocytes entering maternal circulation in deliveries by caesarean section $^{17}$, stillborn deliveries ${ }^{15}$, traumatic vaginal deliveries, deliveries with multiple births, deliveries with signs of premature separation of the placenta, deliveries with pathology in the third stage of labour, etc.

The goal of further studies should be establishing optimal doses of IgG anti-D. The effectiveness of immediate administration of lower doses of IgG anti-D in combination with screening of the volume of fetomaternal hemorrhage and subsequent supplementation of Ig $G$ anti-D in case of necessity should be compared with the effectiveness of administering a single larger dose of $\operatorname{Ig} G$ anti-D to everyone. 
If the amount of fetal erythrocytes which entered the maternal circulation is quantitatively determined, administration of $10 \mu \mathrm{g}$ IgG anti-D per $0.5 \mathrm{ml}$ of fetal erythrocytes or $1 \mathrm{ml}$ of whole blood is indicated ${ }^{6-7}$.

In cases where prevention of $\mathrm{RhD}$ alloimmunization is not performed within 72 hours of a potentially sensitising event, it is still sensible to administer IgG anti-D within 13 days, and in special cases, administration is still recommended up to a maximum interval of 28 days postpartum ${ }^{8}$.

It is necessary to issue a confirming document to women who received $\operatorname{IgG}$ anti-D, which precisely describes the amount and form of administration. IgG anti-D should not be administered to women where the presence of anti-D antibodies was confirmed in their blood serum. Exceptions are cases of persisting levels of antenatally administered IgG anti-D. If there is not absolute certainty regarding the origin of anti-D antibodies in the mother's serum, prevention of RhD alloimmunization should be performed. IgG anti-D should also be administered in cases where the RhD status of the child is unknown.

\section{DETERMINING THE VOLUME OF FETOMATERNAL HEMORRHAGE (FMH)}

The amount of fetal erythrocytes leaked into the maternal circulation (FMH) is accurately assessed using flow cytometry. After labour, a blood sample from the mother may be drawn no sooner than 1 hour after labour and a sample of $0.5-1.0 \mathrm{ml}$ of venous blood is collected into a test tube containing an anticoagulative substance (EDTA, Heparin).

It is common practise to always examine the RhD status of a child born to an RhD negative woman after labour. Assessing the volume of fetomaternal hemorrhage $(\mathrm{FMH})$ is then indicated in cases, where the child is $\mathrm{RhD}$ positive as it allows specification of the dose of IgG anti-D needed for the prevention of RhD alloimmunization of the mother. IgG anti-D is however always administered in a minimally $100 \mu \mathrm{g}$ dose immediately after detecting a positive RhD status of the child.

Optimal and economically most effective would be to apply a $250 \mu \mathrm{g}$ dose of anti-D antibody in the $28 \mathrm{th}$ week of gestation to RhD-negative women if no anti-D antibodies were detected in their serum. After the birth of an RhD positive child, the volume of fetal erythrocytes which entered maternal circulation should be assessed and only in indicated cases should a dose of IgG anti-D be administered postpartum (Australia) ${ }^{4}$.

The volume of fetomaternal hemorrhage (FMH) should also be determined in all $\mathrm{RhD}$ negative women where the presence of anti-D antibodies was not determined, in cases of potentially sensitising events (Table 1) after 20 weeks of gestation ${ }^{5-7,20-23}$.

The Kleihauer-Betke acid-elution test (UK) or the Rosette test (USA) may be used to roughly determine the volume of fetomaternal hemorrhage.

\section{ESTABLISHING RHD GENOTYPE OF THE FETUS}

At the beginning of pregnancy of an RhD negative woman, it is possible to establish the RHD genotype of the fetus from free fetal DNA circulating in maternal peripheral blood. If the fetus is RhD negative, it is unnecessary to administer IgG anti-D at 28 weeks gestation or perform RhD alloimmunization prevention in cases of potentially sensitising events (Table 1) ) $^{24-27}$.

\section{ACKNOWLEDGEMENTS}

Supported by the grant from the Ministry of Health of the Czech Republic IGA NS 10311-3/2009 "Incidence, volume and risk factors of fetomaternal hemorrhage during labour".

\section{REFERENCES}

1. Martin JA, Hamilton BE, Ventura SJ et al. Births: final data for 2001. Natl Vital Stat Rep 2002; 51: 1-102.

2. ACOG - American College of Obstetricians and Gynecologists. Prevention of RhD alloimmunization. ACOG practice bulletin no.4. Washington, DC, 1999.

3. Fung KFK, Eason E, Crane J et al. Maternal-Fetal Medicine Committee, Genetics Committee. Prevention of Rh alloimmunization. J Obstet Gynaecol Can 2003; 25: 765-773.

4. RANZCOG - Royal Australian and New Zealand College of Obstetricians and Gynaecologists. Guidelines for the use of RH D Immunoglobulin (Anti-D) in obstetrics in Australia 2007. Available from: www.ranzcog.edu.au/publications/statements/Cobs6.pdf

5. RCOG - Royal College of Obstetrics and Gynaecology. United Kingdom. Green Top Guidelines 2002. Use of Anti-D immunoglobulin for Rh prophylaxis 2002. Available from: www.rcog.org.uk/ index.asp?PageID=512

6. Crowther C, Middleton P. Anti-D administration after childbirth for preventing Rhesus alloimmunisation. The Cochrane Database of Systematic Reviews, 2009, 3 (updated November 2008).

7. Crowther C, Middleton P. Anti-D administration in pregnancy for preventing Rhesus alloimmunisation. The Cochrane Database of Systematic Reviews, 2009, 3 (updated November 2008).

8. Bowman JM. Controversies in Rh prophylaxis. Who needs Rh immune globulin and when shoud it be given? Am J Obstet Gynecol 1985; 151: 289-294.

9. Contreras M. The prevention of Rh haemolytic disease of the fetus and newborn - general background. Br J Obstet Gynaecol 1998; 105: 7-10.

10. Urbaniak $\mathrm{S}$. The clinical application of anti-D prophylaxis. In: Hadley A, Soothill P. Alloimmune disorders of pregnancy: anaemia, thrombocytopenia and neutropenia in the fetus and newborn. Cambridge, UK: Cambridge University Press; 2002. p. 97-120.

11. Brambati B, Guercilena S, Bonacchi I et al. Feto-maternal transfusion after chorionic villus sampling: clinical implications. Hum Reprod 1986; 1: 37-40.

12. Augustson BM, Fong EA, Grey DE et al. Postpartum anti-D: can we safely reduce the dose? Med J Aust 2006; 184: 611-613.

13. Moise KJ Jr. Management of rhesus alloimunization in pregnancy. Obstet Gynecol 2002; 100: 600-611.

14. Ness PM, Baldwin ML, Niebyl JR. Clinical high-risk designation does not predict excess fetal-maternal haemorrhage. Am J Obstet Gynecol 1987; 156: 154-158.

15. Sebring E, Polesky H. Fetomaternal hemorrhage: incidence, risk factors, time of occurence and clinical effects. Transfusion 1990; 30: $344-357$. 
16. Stedman C, Baudin J, White C et al. Use of the erythrocyte rosette test to screen for excessive fetomaternal hemorrhage in $\mathrm{Rh}$ negative women. Am J Obstet Gynecol 1986; 154: 1363-1369.

17. Feldman N, Skoll A, Sibai B. The incidence of significant fetomaternal haemorrhage in patients undergoing cesarean section. Am J Obstet Gynecol 1990; 163: 855-858.

18. Chilcott J, Lloyd JM, Wight J et al. A review of the clinical effectiveness and cost effectiveness of routine anti-D prophylaxies for pregnant women who are Rhesus (RhD) negative 2002, National Institute of Clinical Excelence, London.

19. NHMRC - National Health and Medical Research Council. Australia. National Blood Authority. Gudelines on the prophylactic use of Rh D immunoglobulin (anti-D) in obstetrics 2003.

20. RCOG - Royal College of Obstetrics and Gynaecology. United Kingdom. Anti-D Immunoglobulin for Rh Prophylaxis 2002. Available from: http://www.rcog.org.uk/womens-health/clinicalguidance/use-anti-d-immunoglobulin-rh-prophylaxis-green-top-22

21. RCOG - Royal College of Obstetrics and Gynaecology. United Kingdom. NICE - National Institute for Clinical Excellence's Technology Appraisal Guidance No 156. Routine antenatal antiD prophylaxis for women who are rhesus D negative, August 2008 (Expected date of next issue - May 2011).

Available from: http://www.nice.org.uk/nicemedia/pdf/ TA156Guidance.pdf

Available from: http://www.nice.org.uk/TA156
22. Moise KJ Jr. Prevention of Rh (D) alloimmunization. The UpToDate Database of Systematic Reviews 2008 (updated April 2008).

Available from: www.uptodate.com

23. Moise KJ Jr. Pathogenesis and prenatal diagnosis of Rhesus (Rh) alloimmunization. The UpToDate Database of Systematic Reviews 2008 (updated April 2008).

Available from: www.uptodate.com

24. Kumar S. Universal RHD genotyping in fetuses. BMJ 2008; 336: 783-4.

25. Finning K, Martin P, Summers J, Massey E, Poole G, Daniels G. Effect of high throughput RHD typing of fetal DNA in maternal plasma on use of anti-RhD immunoglobulin in $\mathrm{RhD}$ negative pregnant woman: prospective feasibility study. BMJ 2008; 336: 816-8.

26. Szczepura A, Bonsel G, Krauth Ch, Osipenko L, Haverkamp A. Is fetal RHD typing in all RhD negative women cost effective? BMJ 2008; 336: 906.

27. Daniels G, Finning K, Martin P, Massey E. Noninvasive prenatal diagnosis of fetal blood group phenotypes: current practice and future prospects. Prenat. Diagn. 2009; 29: 101-107.

28. INTERNATIONAL FORUM: Engelfriet CP, Reesink HW, Judd WJ, Ulander VM, Kuosmanen M, Koskinen S, Rouger P, Morelati F, Tantalo V, Fujii T, de Haas M, van der Schoot CE, Overbeeke M, Koelewijn J, Bonsel G, Vrijkotte T, Zupańska B, Martin-Vega C, Parra Lopez R, de Silva M, Contreras M, Panzer S, Ulm B, Mayr WR. Current status of immunoprophylaxis with anti-D immunoglobulin. Vox Sang. 2003; 85: 328-337. 
